\title{
Design, Modeling, and FPAA-Based Control of a High-Speed Atomic Force Microscope Nanopositioner
}

\author{
Yuen Kuan Yong, Member, IEEE, Bharath Bhikkaji, Member, IEEE, and S. O. Reza Moheimani, Fellow, IEEE
}

\begin{abstract}
An $X Y Z$ nanopositioner is designed for fast the atomic force microscopy. The first resonant modes of the device are measured at 8.8, 8.9, and $48.4 \mathrm{kHz}$ along the $X$-, $Y$-, and $Z$-axes, respectively, which are in close agreement to the finite-element simulations. The measured travel ranges of the lateral and vertical axes are $6.5 \mu \mathrm{m} \times 6.6 \mu \mathrm{m}$ and $4.2 \mu \mathrm{m}$, respectively. Actuating the nanopositioner at frequencies beyond $1 \%$ of the first resonance of the lateral axes causes mechanical vibrations that result in degradation of the images generated. In order to improve the lateral scanning bandwidth, controllers are designed using the integral resonant control methodology to damp the resonant modes of the nanopositioner and to enable fast actuation. Due to the large bandwidth of the designed nanopositioner, a field programmable analog array is used for analog implementation of the controllers. Highresolution images are successfully generated at $200-\mathrm{Hz}$ line rate with $200 \times 200$ pixel resolution in closed loop.
\end{abstract}

Index Terms-Field-programmable analog array (FPAA), flexure-guided positioners, high-speed atomic force microscope (AFM), integral resonant control (IRC), nanopositioner, piezoelectric actuators.

\section{INTRODUCTION}

$\mathbf{F}$ LEXURE-BASED nanopositioners have been used as precision instrumentation in many industrial and research fields, including micro-/nanomanipulation [1]-[4], mirror steering platforms [5], nanoimprint lithography [6], [7], nanometrology [8] and nanomanufacturing [9]. A very important emerging application for flexure-guided, piezoelectric stack driven nanopositioners is in high-speed and video-rate atomic force microscopes (AFM) [10]-[13]. This is due to the high mechanical bandwidth and low cross-coupling among various motion axes of these nanopositioners.

In an AFM, the sample, for which a topographic map is desired, is placed on a XYZ nanopositioner. A cantilever with a sharp probe is placed in contact with the sample. A laser source

Manuscript received March 11, 2011; revised July 8, 2011 and November 3, 2011; accepted March 27, 2012. Date of publication May 11, 2012; date of current version January 18, 2013. Recommended by Technical Editor Y. Sun. This research was supported by the Australia Research Council.

Y. K. Yong and S. O. R. Moheimani are with the School of Electrical Engineering and Computer Science, The University of Newcastle, Callaghan, N.S.W. 2308, Australia (e-mail: yuenkuan.yong@ @ewcastle.edu.au; reza.moheimani@newcastle.edu.au).

B. Bhikkaji is with the Department of Electrical Engineering, Indian Institute of Technology Madras, Chennai 600036, India (e-mail: bharath.bhikkaji@iitm.ac.in).

Color versions of one or more of the figures in this paper are available online at http://ieeexplore.ieee.org.

Digital Object Identifier 10.1109/TMECH.2012.2194161 is focused on the probe end of the cantilever, and the reflection from the cantilever is captured by a position sensitive photo diode (PSPD). When operating the AFM in a constant force contact mode, the cantilever is slowly landed on the sample. Landing causes the cantilever to deflect and the deflection is measured by the PSPD. The output of the PSPD is then compared with a prescribed value. The nanopositioner is moved along the $Z$ direction through a vertical feedback controller to set the cantilever deflection such that the PSPD output equals the prescribed value. After landing, the nanopositioner is moved in a raster pattern. For constant force contact mode, the vertical feedback controller continues regulating the deflection of the cantilever during scanning, thus maintaining the probe-sample interaction force. A topographic image of the sample is derived from the feedback signal generated by the controller.

To generate a raster signal for scanning, one of the lateral axes of the nanopositioner is forced to track a fast triangular waveform, while the other is required to track a slowly increasing ramp, or a pseudo staircase signal. Harmonic components of the triangular waveform with their frequency contents near the resonance frequency of the nanopositioner will induce vibration in the structure [14], which in turn introduce artifacts on the scanned images [15]. Therefore, the scan rate of a nanopositioner is typically restricted to $1 \%$ of the first resonance frequency [16]. To illustrate this, the scan rate of a nanopositioner with a $200-\mathrm{Hz}$ first resonant mode is limited to $2 \mathrm{~Hz}$. For an image of $256 \times 256$ pixel resolution, this equates to more than 2 min to acquire an image. This is clearly inadequate for applications such as monitoring fast-paced biological cell events [10].

The scan speed of an AFM is also limited by the vertical feedback bandwidth that regulates the probe-sample interaction force during scanning [17], [18]. It is shown in [18] that the maximum vertical feedback bandwidth with an integral controller is limited by the first $Z$-axis resonance frequency of the nanopositioner. A nanopositioner system with an inadequate vertical feedback bandwidth is not fast enough to track sharp features, which leads to the "smudging" effect of the feature edges [19].

This paper reports the design of a parallel-kinematic nanopositioner with a large lateral $(X$ - and $Y$ - axes) and vertical $(Z$ axis) mechanical bandwidth. High-bandwidth serial-kinematic nanopositioners have been developed for AFM imaging [10], [15]. A disadvantage of a serial-kinematic nanopositioner is that high mechanical bandwidth can only be achieved in one lateral axis. This is mainly due to the relatively heavy weight that the base actuator would have to carry. Another disadvantage is the inability to measure and correct for parasitic motions 
caused by the cross-coupling effect from other axes [15]. A highspeed parallel-kinematic nanopositioner was presented in [20] and [21]. This nanopositioner has the first lateral resonance frequency of $2.7 \mathrm{kHz}$ that is about three times lower than that presented in this paper. Another high-speed parallel-kinematic nanopositioner was presented in [11]. The nanopositioner has high resonance frequencies, but it contains nine piezoelectric stack actuators. Costly high-power, high-bandwidth piezoamplifiers are required to drive the actuators. The parallel $X Y Z$ nanopositioner presented in this paper only requires three piezoelectric stack actuators. The cross-coupling effect among the three axes is also minimized to avoid image artifacts that may arise due to this effect. Furthermore, the nanopositioner is designed to be of a very small form factor, so that it may be used with AFM that are designed to work with piezoelectric tube scanners.

The scan rate of the nanopositioner can be further increased by suppressing the first lateral resonant mode of the nanopositioner in both $X$ - and $Y$-axes. A number of efficient controllers have been designed for and successfully implemented on nanopositioning stages in recent years, including resonant controllers [22], positive position feedback controllers [23], [24], integral resonant controllers (IRC) [25] and piezoelectric shunt controllers [26], [27]. The IRC, which is a high-performing controller that provides significant damping to resonant modes, have been used to suppress vibration of nanopositioning devices [21], [25], [28]. These nanopositioning devices have resonant modes of less than $3 \mathrm{kHz}$. As a result, the IRC can be implemented digitally, e.g., using a DSP-based rapid prototyping system. Such prototyping systems have restricted sampling rates, e.g., dSPACE-1103 can operate at sampling rates as high as $80 \mathrm{kHz}$. In the case of high bandwidth nanopositioners, such as the one designed here, the limitation in sampling rate makes it impossible to implement the requisite controllers on a typical rapid prototyping systems. Thus, a field programmable analog array (FPAA) is used to implement the required controller. This amounts to a direct analog controller implementation, which allows for a much higher control bandwidth [29]. Also, the IRC in this paper has been extended to accommodate time delay in the system that has not been previously discussed in [21], [25], and [28].

The remainder of the paper is organized as follows. In Section II, the design of the nanopositioner is described. This is followed by the derivation of analytical equations for stiffness and resonance frequency estimations and a discussion of the finite-element (FE) model that was generated in ANSYS to analyze the design. The electrical aspects of the piezoelectric stack actuators are also discussed there. Section III presents the characterization of the nanopositioner where its frequency responses and the cross-couplings associated with the three axes are measured and discussed. Section IV presents the details of the IRC design and implementation. The controlled nanopositioner is integrated with a commercial NT-MDT NTEGRA AFM to demonstrate its scan performance. The imaging performance of the nanopositioner is presented in Section V. Finally, Section VI concludes the paper.

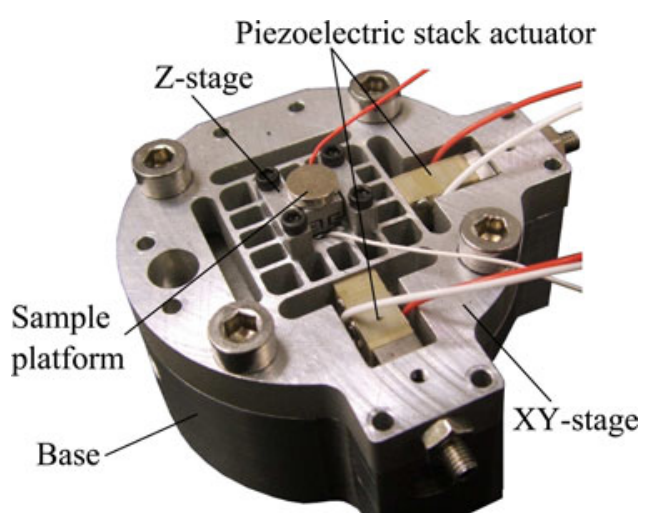

(a)

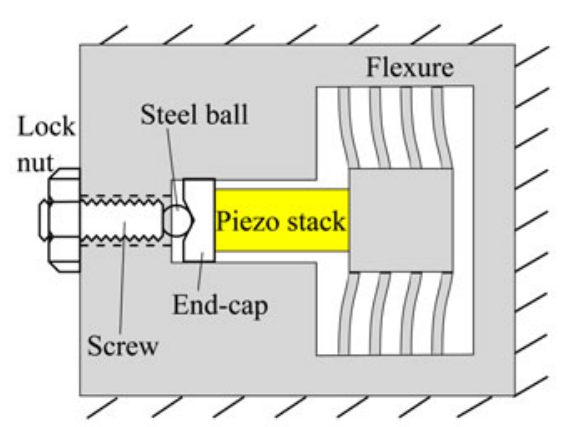

(b)

Fig. 1. (a) First $X Y Z$ nanopositioner design. (b) Preload mechanism.

\section{High-SPEEd FleXURE-BASEd XYZ NANOPOSITIONER DESIGN}

The flexure-based nanopositioner proposed in this paper has a parallel-kinematic configuration. Due to its mechanical symmetry in $X-Y$ plane, the nanopositioner can be operated in both raster and nonraster scan modes [30], [31] [32]. The device consists of a $X-Y$ nanopositioning stage and a $Z$ nanopositioning stage mounted onto the $X-Y$ platform. A piezoelectric stack actuator is used to drive each motion axis of the nanopositioner. For the $X-Y$ nanopositioner, left-spring or beam-like flexures are used to guide the sample stage along the direction of motion of the actuator, while restraining other parasitic motions.

A previous version of the proposed design, also reported in [12], is shown in Fig. 1(a). It has a displacement range of $4,4.5$, and $4 \mu \mathrm{m}$ in the $X, Y$, and $Z$ directions, respectively. The first resonance frequencies of the $X$-, $Y$-, and $Z$-axes were measured to be in the vicinity of $10 \mathrm{kHz}$. The design incorporates a preload mechanism which consists of a steel ball, an end cap with a V-groove, a screw and a lock nut as shown in Fig. 1(b). In this arrangement the preload is applied by tightening the screw, which causes the steel ball to press against the end cap, which in turn aligns the piezoelectric stack with the direction of the applied force.

The $X Y Z$ nanopositioner design proposed here, and pictured in Fig. 2(a), is an improvement on the previous design reported in [12]. It is more compact and the preload mechanism consists of a much simpler mechanism, which uses flexures alone. To preload the $X$ and $Y$ piezoelectric stacks, weights are first 


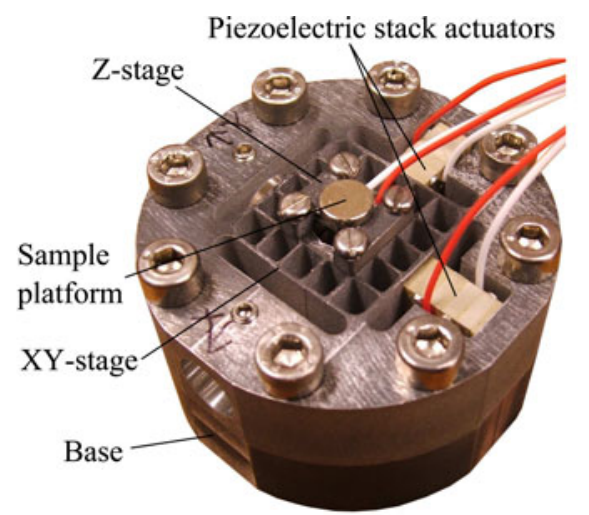

(a)

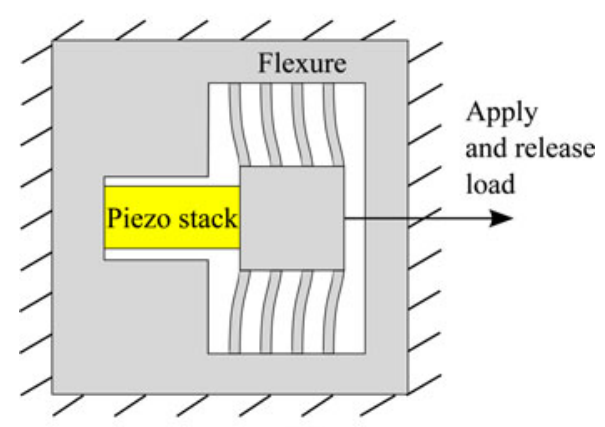

(b)

Fig. 2. (a) Improved $X Y Z$ nanopositioner proposed in this paper. (b) Preload method.

applied to deform the flexure elastically, allowing a piezoelectric stack actuator to be placed in its designated position. Weights are then released to restore the original position of the flexures. The procedure results in the application of the necessary preload to the piezoelectric stack. This preload approach eliminates the need for the screw, steel ball, end-cap and lock nut, thus simplifying the assembly process [15]. To make the preload method as shown in Fig. 2(b) practical, the thickness of each flexure was reduced by $40 \%$. This ensures that the flexures can be elastically deformed when the weights are applied. Due to the reduction of flexure stiffness, the resonance frequencies of the proposed nanopositioner were slightly decreased but not significant. The measured first mechanical resonance frequency of both the $X$ and $Y$-axes are 8.9 and $8.8 \mathrm{kHz}$, respectively. The design of the Z-nanopositioner was also improved to have a much lower profile. Thus, the $Z$-nanopositioner is much less susceptible to unwanted "rocking" modes, when operated at high speeds. The maximum $X, Y$, and $Z$ displacements of the nanopositioner were measure to be $6.5,6.6$, and $4.2 \mu \mathrm{m}$, respectively, which again an improvement over the previous design.

The $Z$-axis resonant mode of the proposed nanopositioner appears at $48.4 \mathrm{kHz}$, which is nearly 5 times that of the previous design. When imaging smooth features, the previous design provides good quality topographic images at high scan speeds [33]. However, it is inadequate for imaging sharp sample features because the vertical feedback controller, which is limited by the lowest resonant mode of the $\mathrm{Z}$-axis, is too slow to track such features [19]. The nanopositioner proposed in this paper has a
TABLE I

NOTATION

\begin{tabular}{|ll|}
\hline$t$ & Beam thickness \\
$L$ & Beam length \\
$b$ & Beam depth \\
$A$ & Cross-sectional area, $A=b t$ \\
$E$ & Young's modulus \\
$v$ & Poisson's ratio \\
$G$ & Shear modulus, $G=E /[2(1+v)]$. \\
$I$ & Second moment of inertia about the Z-axis, $I=b t^{3} / 12$ \\
$J$ & Polar moment of inertia, $J=b t\left(b^{2}+t^{2}\right) / 12$. \\
$\alpha$ & Shear correction factor, i.e. $6 / 5$ for rectangular cross-section.
\end{tabular}

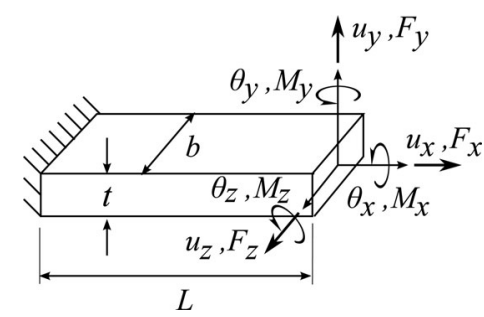

Fig. 3. Beam flexure with its boundary conditions, loadings, and dimensions.

maximum vertical bandwidth of $4.8 \mathrm{kHz}$ (estimated using [18], eq. (8)]). Together with the damping controllers implemented in the $X$ - and $Y$-axes, the proposed nanopositioner can be used to image sharp sample features at scan rates as high as $200 \mathrm{~Hz}$ without notable vibration-induced artifacts in the images.

The following section details of the design procedures that led to this nanopositioner.

\section{A. Displacement and Stiffness Analysis}

A key design specification for a high-speed AFM nanopositioner is a very high first mechanical resonance frequency along the actuation direction [14], [34]. This requirement, however, often leads to a reduction in the travel range of the stage. This is due to the inevitable increase in the structural stiffness when the resonance frequency is increased. This tradeoff is best described by $\omega_{n}=\sqrt{k / m}$, where $k$ and $m$ represent the effective stiffness and mass of the stage, respectively, and $\omega_{n}$ is the resonance frequency of the mechanical system. The following study of the effect of flexure dimensions on the nanopositioner's resonance frequency and displacement was instrumental in reaching a compromise between the speed and travel range of the stage.

The stiffness of individual flexure was derived using strain energy method [35] and Castigliano's second theorem [36], [37]. These methods have been commonly used in the flexure design literature [2], [34], [38], [39]. The in-plane displacement of a beam flexure can be defined as

$$
\left[\begin{array}{l}
u_{x} \\
u_{y} \\
\theta_{z}
\end{array}\right]=\left[\begin{array}{ccc}
C_{x, F_{x}} & 0 & 0 \\
0 & C_{y, F_{y}} & C_{y, M_{z}} \\
0 & C_{\theta_{z}, F_{y}} & C_{\theta_{z}, M_{z}}
\end{array}\right]\left[\begin{array}{c}
F_{x} \\
F_{y} \\
M_{z}
\end{array}\right]
$$

where the displacements and loads are as shown in Fig. 3. The compliance values are derived in [34], as $C_{x, F_{x}}=L / A E, \quad C_{y, F_{y}}=L / 3 E I+\alpha L / G A, \quad C_{y, M_{z}}=$ $C_{\theta_{z}, F_{y}}=L^{2} / 2 E I, C_{\theta_{z}, M_{z}}=L / E I$. The corresponding notation is described in Table I. 


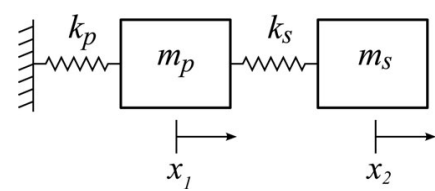

Fig. 4. Each actuation axis of the XYZ nanopositioner is modeled as a 2-DOF undamped system. $m_{p}$ and $k_{p}$ are the mass and stiffness of the piezoelectric stack actuator. $m_{s}$ and $k_{s}$ are the mass and stiffness of the nanopositioning nanopositioner.

For a fixed-guided beam, the resultant moment $M_{z}$ at the guided end of the beam due to the applied $F_{y}$ at that point is $M_{z}=-F_{y} L / 2$. Note that the proposed flexures used in this paper are short flexures. Therefore, the shear-induced deformation term $\alpha L / G A$ in $C_{y, F_{y}}$ should not be ignored. Inclusion of the shear term is known to result in different deformations in flexures [40]. From (1), the displacement of the flexure along the $y$-direction is obtained as

$$
\begin{aligned}
u_{y} & =C_{y, F_{y}} F_{y}+C_{y, M_{z}} M_{z} \\
& =F_{y}\left(\frac{L^{3}}{3 E I}+\frac{\alpha L}{G A}\right)-\frac{F_{y}}{2}\left(\frac{L^{3}}{2 E I}\right) .
\end{aligned}
$$

Substituting $I=b t^{3} / 12$ and $A=b t$ into (2), and rearranging the equation, the stiffness of the flexure along the $y$-direction is determined as

$$
k_{y}=\frac{F_{y}}{u_{y}}=\left[\frac{1}{E b}\left(\frac{L}{t}\right)^{3}+\frac{\alpha}{G b}\left(\frac{L}{t}\right)\right]^{-1} .
$$

Since we know $E, G$, and $b$ the parameter that determines the flexure stiffness is $L / t$. The flexures of the $X Y Z$ nanopositioner in Fig. 2 are arranged in parallel. Therefore, the general stiffness equation of the $X Y Z$ nanopositioner along their direction of actuation is $k_{s}=N k_{y}$, where $N$ is the number of flexures. The maximum displacement of the nanopositioner $D_{s}$ is dependent on the piezoelectric stack actuator and it is estimated as

$$
D_{s}=\Delta l\left(\frac{k_{p}}{k_{p}+k_{s}}\right)
$$

where $\Delta l$ is the maximum stroke of the unloaded piezoelectric stack actuator, and $k_{p}$ is the stiffness of the piezoelectric actuator.

\section{B. Resonance Frequency Estimation}

The $X Y Z$ resonance frequencies along the direction of actuation are estimated using the algebraic eigenvalue method [41]. Assuming the system is a 2-DOF undamped system as shown in Fig. 4, we may write

$$
\mathbf{M} \ddot{\mathbf{x}}+\mathbf{K x}=\mathbf{0}
$$

where $\mathbf{M}=\left[\begin{array}{cc}m_{p} & 0 \\ 0 & m_{s}\end{array}\right]$ and $\mathbf{K}=\left[\begin{array}{cc}k_{p}+k_{s} & -k_{s} \\ -k_{s} & k_{s}\end{array}\right] . m_{p}$ and $k_{p}$ are the mass and stiffness of the piezoelectric stack actuator. Also, $m_{s}$ and $k_{s}$ are the mass and stiffness of the nanopositioner. Substituting $\mathbf{x}=\mathbf{M}^{-1 / 2} \mathbf{q}$ into (5) and multiplying the resulting equation by $\mathbf{M}^{-1 / 2}$ yields

$$
\mathbf{I} \ddot{\mathbf{q}}(t)+\mathbf{M}^{-1 / 2} \mathbf{K M}^{-1 / 2} \mathbf{q}(t)=\mathbf{0} .
$$

Let $\widetilde{\mathbf{K}}=\mathbf{M}^{-1 / 2} \mathbf{K M}^{-1 / 2}$, by substituting $\mathbf{q}(t)=\mathbf{v} e^{j \omega t}$, where $\mathbf{v}$ is a constant vector, into (6) we obtain

$$
\widetilde{\mathbf{K}} \mathbf{v}=\omega^{2} \mathbf{v} \text {. }
$$

If we replace $\omega^{2}$ with $\lambda$ in (7), the solutions for $\lambda$ are the eigenvalues of the matrix $\widetilde{\mathbf{K}}$. The resonance frequencies are $f_{1}=\sqrt{\lambda_{1}} / 2 \pi$ and $f_{2}=\sqrt{\lambda_{2}} / 2 \pi$. Note that the smaller of the two frequencies $f_{1}$ and $f_{2}$ will be the first translational mode of the system and is denoted as $f_{\text {res }}$.

\section{Parametric Study}

In this section, a parametric study of the $X Y$ nanopositioner design is presented. The $Z$ nanopositioner was designed using identical principles. Thus, its analysis is not be presented for brevity. In order to have a quantitative analysis of the $X Y$ nanopositioner design, the relationship among $N, L / t, k_{s}, D_{s}$ and $f_{\text {res }}$ are plotted in Fig. 5. The number of flexures $N$ is stepped from 1 to 20 and $L / t$ is varied from 3 to 15 .

As the ratio of $L / t$ increases, the stiffness and resonance frequency decrease while the displacement increases. This is an unavoidable tradeoff between stiffness/resonance frequency and displacement. Intuitively, the resonance frequency increases when the number of flexures $N$ increases. However, the additional mass of each flexure lowers the resonance frequency. For example, as shown in Fig. 5(c), at $L / t=6$ and $N=10, f_{\text {res }}$ is increased to $12 \mathrm{kHz}$, which is a three-fold increment in resonance frequency compared to that of a single flexure $(N=1)$. However, when $N$ is increased to $20, f_{\text {res }}$ is improved only by a factor of four at the expense of the displacement range being reduced by $20 \%$. Therefore, the resonance frequency of the nanopositioner may not be increased significantly by introducing more flexures into the design.

For the $X Y$ nanopositioner design, $K_{s}$ was set to approximately $10 \mathrm{~N} / \mu \mathrm{m}$ to ensure that an adequate preload was applied to the piezoelectric stack actuator. To achieve approximately $10-\mathrm{kHz}$ resonance frequency without sacrificing too much displacement, $L / t=9$ and $N=12$ were selected in the design. For the $Z$ nanopositioner design, $L / t=6$ and $N=4$ were chosen to achieve approximately $50-\mathrm{kHz}$ resonance frequency.

\section{Finite-Element Analysis (FEA)}

The analytical equations derived in Sections II-A and II-B were based on a single axis mass-spring model. They serve as a useful tool for initial parametric design study of the nanopositioner. In this section, a FE model of the $X Y Z$ nanopositioner is presented. The FE simulations were used to obtain a better estimation of the stiffness and resonance frequencies of the nanopositioner.

Meshing was first generated using ANSYS "automesh" tool. Meshing near the flexure was then refined manually to obtain more precise results. The material of the nanopositioner is aluminum alloy Al7075 with Young's modulus of $72 \mathrm{GPa}$. For 


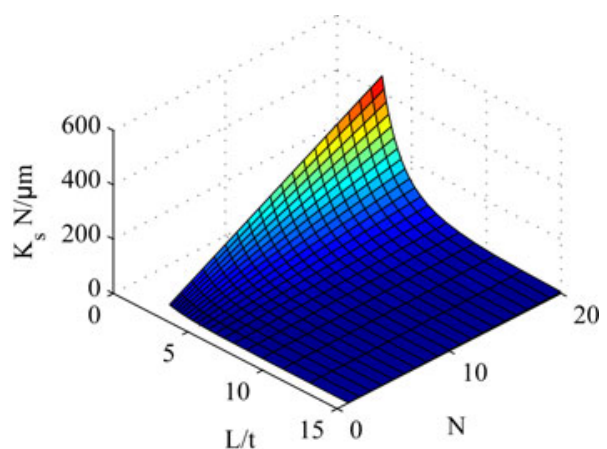

(a)

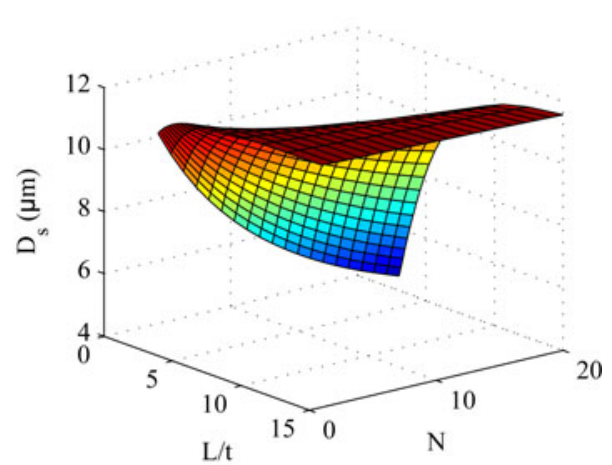

(b)

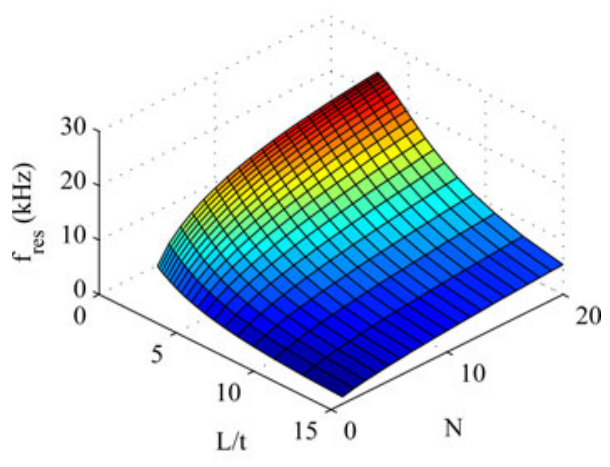

(c)

Fig. 5. Displacement $D_{s}$, stiffness $K_{s}$, and resonance frequency $f_{\text {res }}$ plots of the XYZ nanopositioner in relation to the ratio $L / t$ and the number of flexure $N$.

modal analysis, motions of the base, in which the $X Y$ nanopositioner was attached, were fixed during simulation. For the $Z$ nanopositioner, the motions of the $X Y$ nanopositioner were fixed to simplify the analysis. The simulated first resonant frequencies of the stage along the $X$ - and $Y$-axes both are at $10.7 \mathrm{kHz}$. For the $Z$-axis, the dominant mode occurs at $52.8 \mathrm{kHz}$. FE simulations of the stage are illustrated in Fig. 6.

For stiffness analysis, a point force was applied to the $X Y Z$ nanopositioner as shown in Fig. 6(d) and (e), and the corresponding displacement of the nanopositioner was recorded. The stiffness is calculated as force over displacement. The simulated stiffness are $13.1 \mathrm{~N} / \mu \mathrm{m}$ for the $X$ - and $Y$-axes, and $5.8 \mathrm{~N} / \mu \mathrm{m}$ for the $Z$-axis.

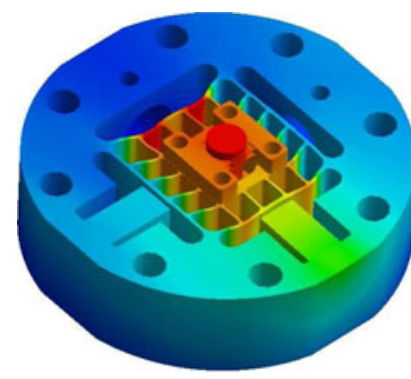

(a)

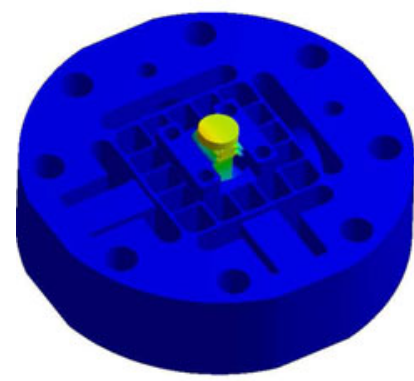

(c)

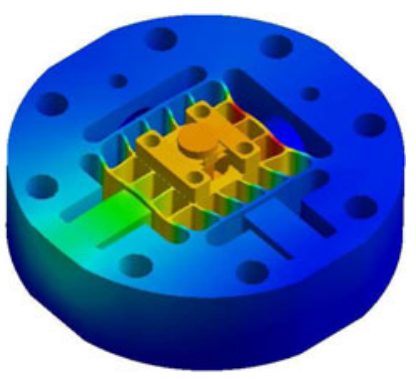

(b)

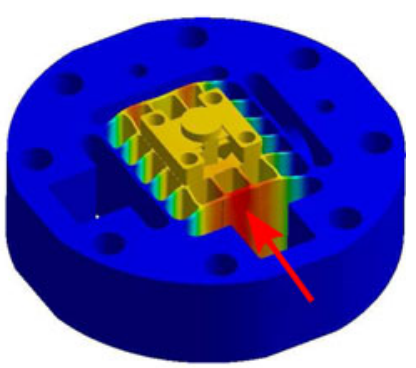

(d)

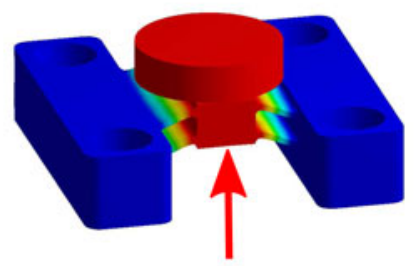

(e)

Fig. 6. FE simulated first mechanical resonance modes along the actuation direction of the $X Y Z$ nanopositioner. (a) $X$-axis: $10.7 \mathrm{kHz}$. (b) $Y$-axis: $10.7 \mathrm{kHz}$. (c) $Z$-axis: $52.8 \mathrm{kHz}$. (d) and (e) Stiffness estimation of the $X Y$ and $Z$ scanners. Force was applied as shown and the corresponding displacement was recorded.

TABLE II

SPECIFICATIONS OF THE PIEZOELECTRIC STACK ACTUATORS

\begin{tabular}{cccccc}
\hline Piezo & $\begin{array}{c}\text { Dimension } \\
\mathrm{mm}\end{array}$ & $\begin{array}{c}\text { Capa. } \\
\mathrm{nF}\end{array}$ & $\begin{array}{c}\text { Stroke @ 200V } \\
\mu \mathrm{m}\end{array}$ & $\begin{array}{c}\text { Stiff. } \\
\mathrm{N} / \mu \mathrm{m}\end{array}$ & $\begin{array}{c}\text { Res. freq. } \\
\mathrm{kHz}\end{array}$ \\
\hline $\mathrm{X}, \mathrm{Y}$ & $5 \times 5 \times 10$ & 380 & 11.8 & 283 & 220 \\
$\mathrm{Z}$ & $3 \times 3 \times 6$ & 70 & 5.5 & 60 & 210 \\
\hline
\end{tabular}

\section{E. Piezoelectric Stack Actuators}

Driving piezoelectric stack actuators at high frequencies amounts to a significant challenge. It is well-known that the larger the capacitance of a piezoelectric stack actuator, the larger the current required to drive the actuator at high speed [13]. The required current may exceed the current limit of the driving amplifier. Thus, the piezoelectric stack actuators must be chosen carefully to ensure their suitability for the specific system. The piezoelectric stack actuators selected to drive the stage along the $X$ and $Y$ directions are Noliac SCMAP07. These actuators have a capacitance of $380 \mathrm{nF}$. The $Z$-axis piezoelectric stack actuator is SCMAP06 that has a capacitance of $70 \mathrm{nF}$. Table II contains detailed specifications of these actuators. Three PiezoDrive PDL200 amplifiers were used to drive the piezos. The maximum operating frequency of driving a triangular signal can be estimated as $f^{3 \mathrm{~dB}}=0.1 / 190 C$ [42]. With the setup 

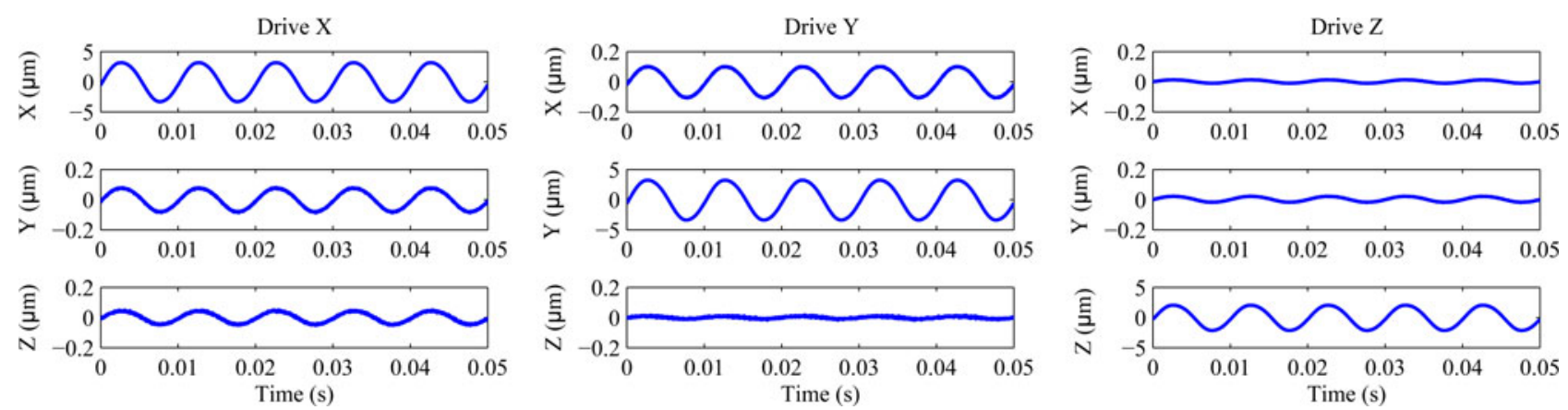

Fig. 7. Displacements and cross-coupling motions of the XYZ nanopositioner.
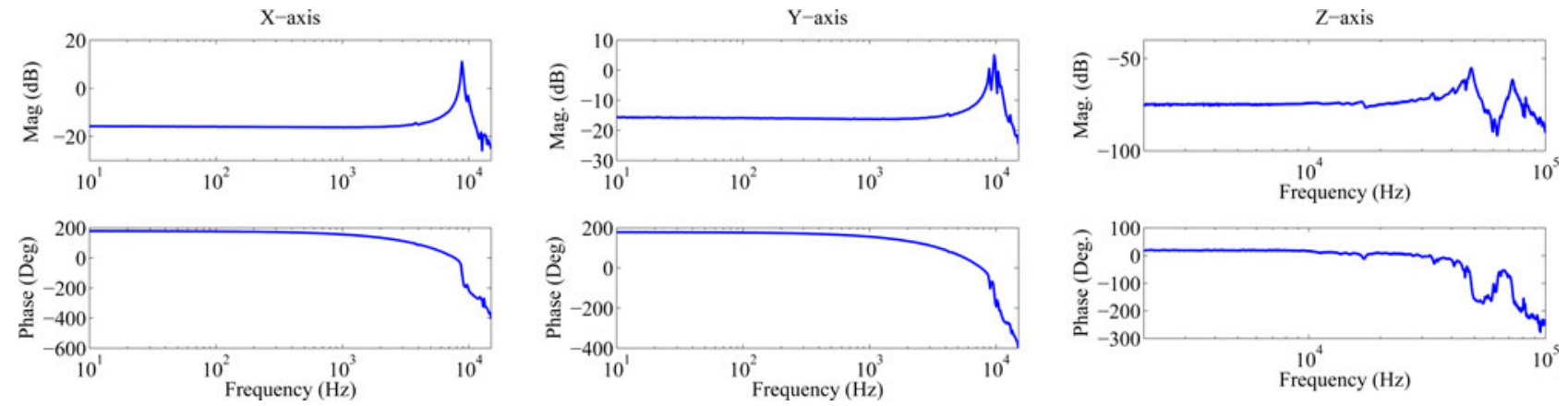

Fig. 8. Measured frequency responses. $X$-axis: The first dominant peak appears at $8.8 \mathrm{kHz} . Y$-axis: The first dominant peak appears at $8.9 \mathrm{kHz}$. $Z$-axis: The first dominant peak appears at $48.4 \mathrm{kHz}$.

described earlier, it is estimated that the fast lateral axis of the nanopositioner can be driven with a triangular signal up to $1.4 \mathrm{kHz}$, at the full voltage range of $200 \mathrm{~V}$, without exceeding the current limit of the amplifier.

\section{CHARACTERIZATION OF THE XYZ NANOPOSITIONER}

To evaluate the displacement range and coupling between various axes of the nanopositioner, a $100-\mathrm{Hz}$ sine-wave of $200 \mathrm{~V}$ was applied to each of the piezoelectric stack actuators. Displacements and cross-coupling motions of the nanopositioner were measured using the ADE Technologies 8810 capacitive sensors and results are plotted in Fig. 7. The measured full displacement range of the $X-, Y_{-}$, and $Z$-axes are 6. 5, 6.6, and $4.2 \mu \mathrm{m}$, respectively. When the $X$-axis is driven by the $200-\mathrm{V}$ sine-wave, the induced cross-coupling motions at the $Y$ and $Z$-axes are $2.5 \%(-31.8 \mathrm{~dB})$ and $1.58 \%(-36 \mathrm{~dB})$ of the $X$ displacement, respectively. When the $Y$-axis is driven, it induces $3.1 \%(-29.9 \mathrm{~dB})$ and $0.53 \%(-45.5 \mathrm{~dB})$ in the $X$ and $Z$ displacement, respectively. By applying the sine-wave to the $Z$-axis, it induces $0.58 \%(-44.7 \mathrm{~dB})$ and $0.99 \%(-40 \mathrm{~dB})$ in the $X$ and $Y$ displacement, respectively. Although these couplings are quite small, they can be made even smaller by using a feedback control technique, such as that reported in [20].

Frequency responses of the $X$ - and $Y$-axes of the nanopositioner were measured using a dual channel spectrum analyzer (HP 35670A). A Microscope Scanning Vibrometer (MSV-400 Polytec) was used to measure the frequency responses of the $Z$ axis. Fig. 8 plots the frequency response functions of the three axes. The first dominant mode for $X_{-}, Y_{-}$, and $Z$-axes are 8.8,
8.9, and $48.4 \mathrm{kHz}$, respectively. Note that the second peak in the $Z$-axis frequency response is the rocking mode of the structure that appears at $78 \mathrm{kHz}$. This nanopositioner is designed so that this rocking mode appears after the $Z$-axis actuation mode. This is to ensure that the presence of rocking mode will not complicate the task of control design [15]. The measured resonance frequencies are within the $20 \%$ range of those predicted by FEA. This is most likely due to the machining errors when the nanopositioner was being manufactured, and the variations in stiffness of piezoelectric stack actuators. Nevertheless, the measured resonances are in reasonable agreement with the FE simulations.

\section{Modeling, Control Design, And Implementation}

As the nanopositioner is to be actuated in a raster pattern along the $X Y$ plane, the device is interpreted as a linear system with two inputs and two outputs

$$
Y_{d}(s)=G_{d}(s) U(s)
$$

where $Y_{d}(s)$ is the Laplace transform of $\left[d_{X}, d_{Y}\right]^{\top}$, displacement measurements of the capacitive sensors along the $X$ - and $Y$-axes, $U(s)$ is the Laplace transform of $\left[v_{X}, v_{Y}\right]^{\top}$, voltage signals applied to electrodes of the piezoelectric stacks along $X$ and $Y$ directions, and

$$
G_{d}(s)=\left[\begin{array}{ll}
G_{\mathrm{XX}}(s) & G_{\mathrm{XY}}(s) \\
G_{\mathrm{YX}}(s) & G_{\mathrm{YY}}(s)
\end{array}\right]
$$

are the transfer-functions relating the inputs $\left[v_{X}, v_{Y}\right]^{\top}$ and the outputs $\left[d_{X}, d_{Y}\right]^{\top}$, respectively. The dashed plots (- -) in Figs. 9 
(a)

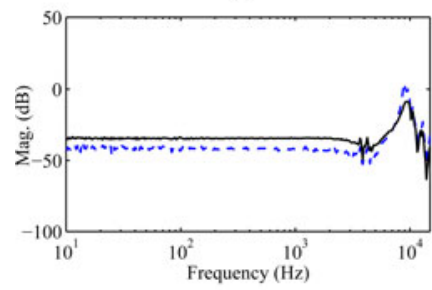

(c)

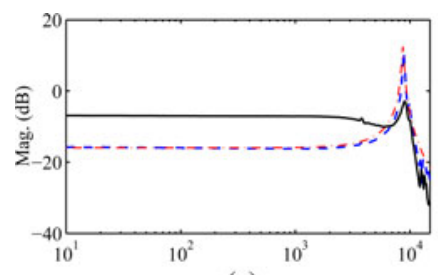

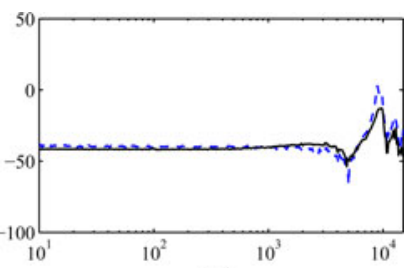

(b)

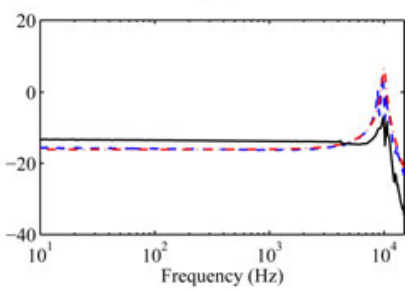

(d)
Fig. 9. Magnitude responses of (a) $d_{X}(i \omega) / v_{X}(i \omega)$, (b) $d_{X}(i \omega) / v_{Y}(i \omega)$, (c) $d_{Y}(i \omega) / v_{X}(i \omega)$, and (d) $d_{Y}(i \omega) / v_{Y}(i \omega)$, where $d_{X}(i \omega), d_{Y}(i \omega), v_{X}(i \omega)$, and $v_{Y}(i \omega)$ denote the Fourier transforms of the corresponding time domain signals. The dashed plots (- -) in (a), (b), (c), and (d) denote the open loop system, dash-dotted plots (---) in (a) and (d) denote the open loop model, while the thick line (-) plots denote the closed-loop response.

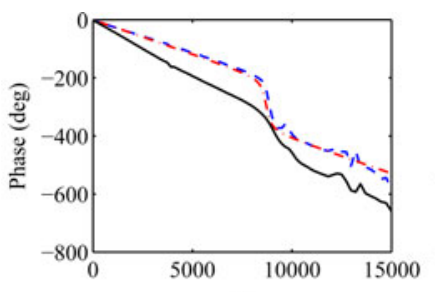

(a)

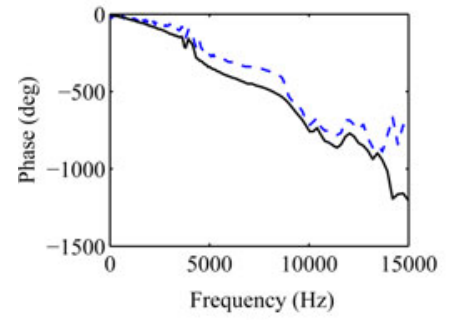

(c)

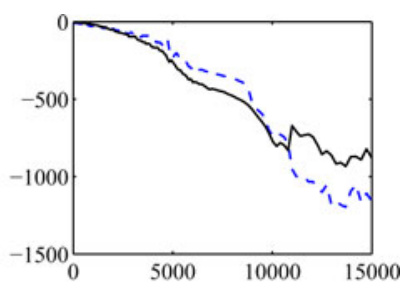

(b)

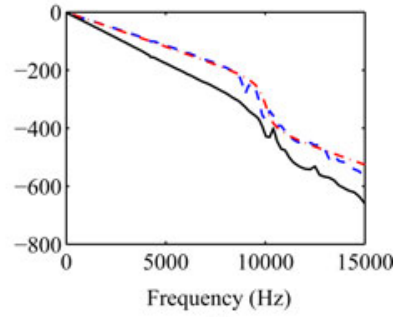

(d)
Fig. 10. Phase responses of (a) $d_{X}(i \omega) / v_{X}(i \omega)$, (b) $d_{X}(i \omega) /$ $v_{Y}(i \omega)$, (c) $d_{Y}(i \omega) / v_{X}(i \omega)$, and (d) $d_{Y}(i \omega) / v_{Y}(i \omega)$, where $d_{X}(i \omega), d_{Y}(i \omega), v_{X}(i \omega)$, and $v_{Y}(i \omega)$ denote the Fourier transforms of the corresponding time domain signals. The dashed plots (- -) in (a)-(d) denote the open loop system, dash-dotted plots (-.-) in (a) and (d) denote the open loop model, while the thick line (-) plots denote the closed-loop response.

and 10 are the magnitude and phase responses of the individual transfer-functions of $G_{d}(s)$, (9). Data for these plots were obtained by applying swept-sine waves along $X$ - and $Y$-axes inputs and recording the corresponding capacitive sensor responses $d_{X}$ and $d_{Y}$. The frequency response functions were obtained by taking Fourier transform of the data set, and computing the respective input to output ratios.

It is apparent from the magnitude response, Fig. 9, that the $X$ and $Y$-axes resonances are roughly between 8.8 and $8.9 \mathrm{kHz}$. In addition to the resonant behavior, the phase response, Fig. 10, appears to include a linear term, suggesting a time delay. In other

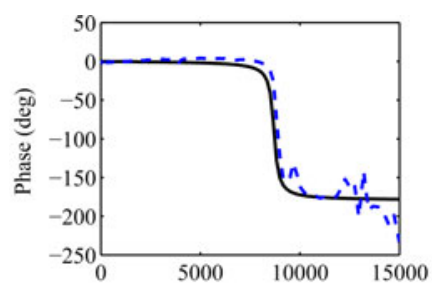

(a)

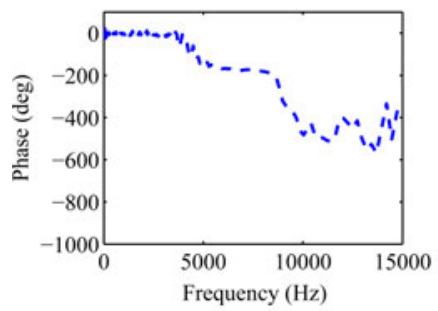

(c)

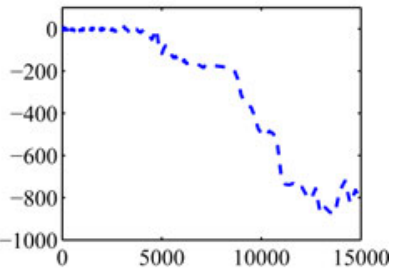

(b)

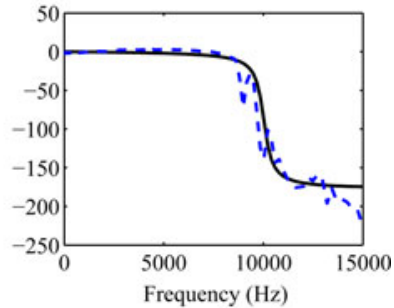

(d)
Fig. 11. Phase responses of (a) $\left(d_{X}(i \omega) / v_{X}(i \omega)\right) e^{\mathrm{i} \omega \tau}$, (b) $\left(d_{X}(i \omega) /\right.$ $\left.v_{Y}(i \omega)\right) e^{i \omega \tau}$, (c) $\left(d_{Y}(i \omega) / v_{X}(i \omega)\right) e^{i \omega \tau}$, and (d) $\left(d_{Y}(i \omega) / v_{Y}(i \omega)\right) e^{i \omega \tau}$, with dashed plots (--) denoting the resonant system and thick plots (-) denoting the corresponding models.

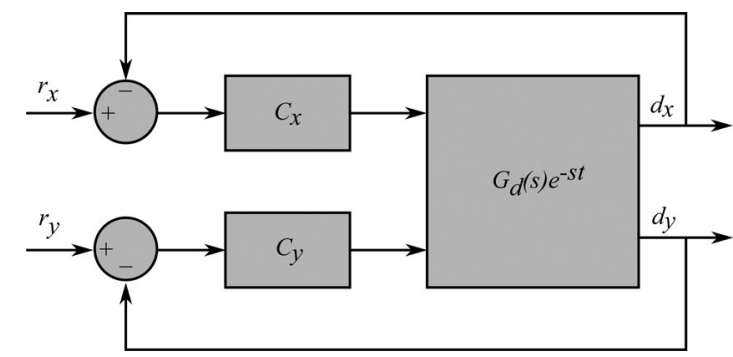

Fig. 12. Feedback configuration.

words, phase plots suggest that the transfer-function matrix is of the form $G_{d}(s) e^{-s \tau}$ for some time delay $\tau>0$, with $G_{d}(s)$ denoting a highly resonant finite order system. The dashed plots (- ) in Fig. 11 are phase responses of the resonant component. These were plotted by estimating the time delay $\tau$ that was found to be approximately $65 \mu \mathrm{s}$, from the phase response presented in Fig. 10, and multiplying the frequency response data by $e^{\mathrm{i} \omega \tau}$. Note that, the time delay $\tau$ is the slope of the straight line joining $0^{\circ}$ to $180^{\circ}$ in the dashed plots in Fig. 10(a) and (d).

Controllers are designed to damp the resonances along both $X$ and $Y$-axes. Here, for controller design only the diagonal terms $G_{X X}(s)$ and $G_{Y Y}(s)$ are considered. The cross-coupling terms $G_{X Y}(s)$ and $G_{Y X}(s)$ are neglected. The feedback configuration corresponding to the control design is presented in Fig. 12. It is apparent from the dash-dotted plots in (a) and (d) of Figs. 9 and 11 that $G_{X X}(s)$ and $G_{Y Y}(s)$ can be satisfactorily modeled as second-order systems. The following models were estimated for the resonant transfer-functions:

$$
G_{X X}(s)=\frac{3.77 \times 10^{8}}{s^{2}+2060 s+2.986 \times 10^{9}}
$$

and

$$
G_{Y Y}(s)=\frac{5.32 \times 10^{8}}{s^{2}+3862 s+3.974 \times 10^{9}} .
$$


The dash-dotted plots in (a) and (d) of Figs. 9 and 11 are the magnitude and phase responses of the models (10) and (11), respectively. As the system also includes a time delay $\tau=65 \mu \mathrm{s}$, the transfer-functions $G_{X X}(s)$ and $G_{Y Y}(s)$ are multiplied by

$$
T(s)=\frac{s^{2}-9.231 \times 10^{4} s+2.84 \times 10^{9}}{s^{2}+9.231 \times 10^{4} s+2.84 \times 10^{9}}
$$

which is a second-order Pade approximation of $e^{-s \tau}$. The phase response of the transfer-functions $G_{X X}(s) T(s)$ and $G_{Y Y}(s) T(s)$ are plotted in Fig. 10(a) and (d). Plots suggest that the models fit the respective data sets with reasonable accuracy.

Approximation of the time delay introduces nonminimum phase zeros. Most literature involving nanopositioners, report the presence of nonminimum phase zeros [14], [43], [44]. The reasons for that have not been satisfactorily explained. It is clear from the aforesaid discussion that, when approximating the nanopositioner dynamics with finite-order models, the time delay present in the system is inadvertently approximated, leading to the presence of nonminimum phase zeros. Most nanopositioners use capacitive sensors to measure the displacement. Frequency response of capacitive sensors closely approximate a Butterworth filter (typically a second-order filter). The phase response of Butterworth filters are approximately linear near the passband region, which causes the time delay.

\section{A. IRC Design and FPAA Implementation}

Smith predictor implementation of controllers negate the effects of time delay in plants [45]. However, the working of Smith predictor is based on the internal model principle [45], which in the current context amounts to cancellation of the highly resonant system poles. This would make the closed-loop system very sensitive to perturbations in system parameters, eventually leading to degradation and nonrepeatability of the scans.

In this paper, IRC is used for damping the resonant modes. Design of IRC using root locus is discussed in detail in [46]. In the current context, for $G_{X X}(s) T(s)$ it amounts to choosing scalars $D_{x}$ and $K_{X}$ such that the zeros of

$$
R_{X X}(s) \triangleq 1+\left(G_{X X}(s) T(s)+D_{X}\right) \frac{K_{X}}{s}
$$

are well inside the left half-plane. The central idea of IRC is to change the zeros by adding a $D_{x}$ so that the root locus of $R_{x x}(s)$, obtained by varying $K_{x}$, lies completely inside the left half-plane. Since

$$
R_{X X}(s)=\frac{s+K_{X} D_{X}}{s}\left(1+G_{X X}(s) T(s) \frac{K_{X}}{s+K_{X} D_{X}}\right)
$$

a controller of the form

$$
C_{X}(s)=\frac{K_{X}}{s+K_{X} D_{X}}
$$

in unity feedback, would have the root locus as (13) for a fixed $D_{X}$. The same procedure holds for $G_{Y Y}(s) T(s)$. Here, it suffices to say that, we have chosen

$$
C_{X}(s)=\frac{4 \times 10^{4}}{s+8 \times 10^{3}}
$$

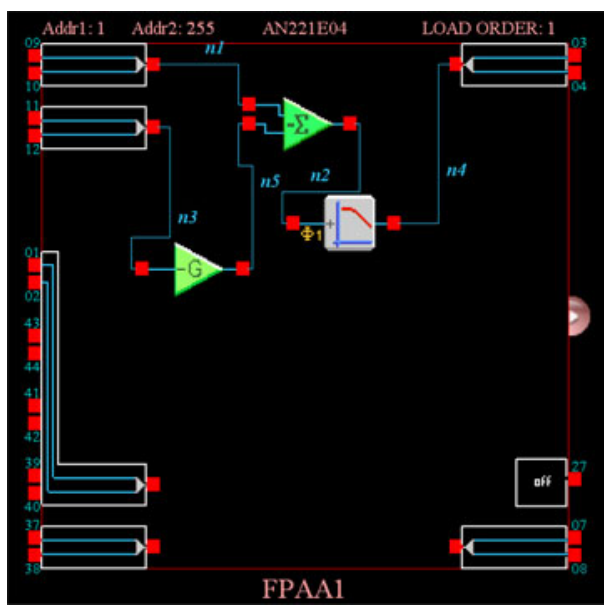

Fig. 13. Implementation of the IRC on the FPAA.

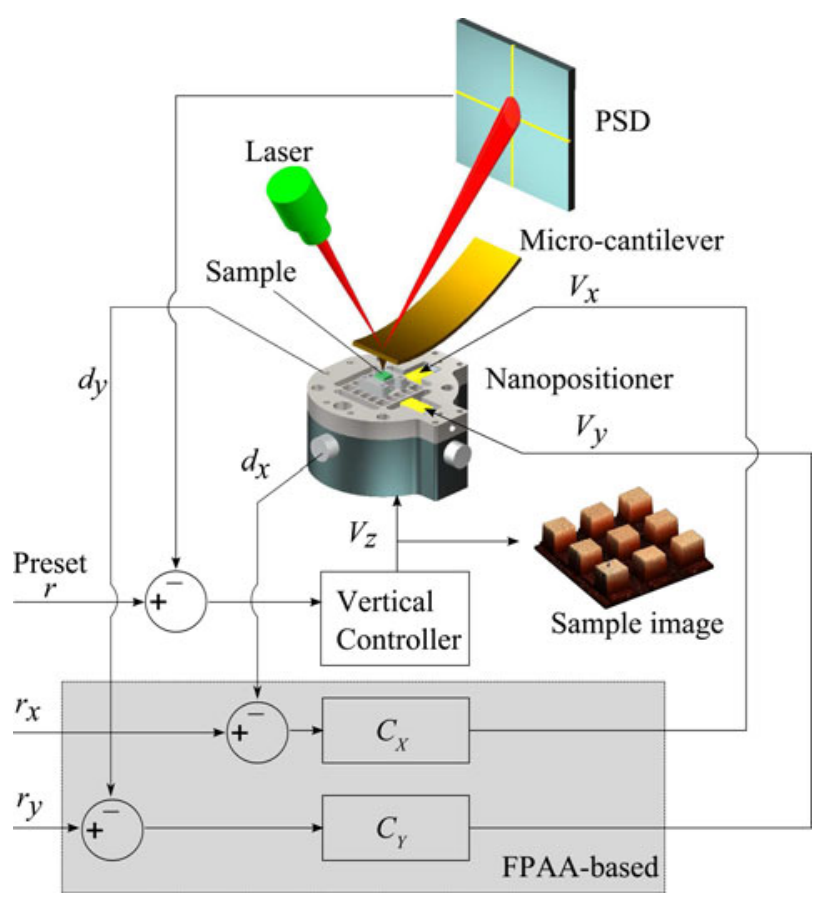

Fig. 14. Constant force contact mode AFM. The $X$ - and $Y$-axes resonant modes are damped using FPAA-based controllers. The vertical feedback is the in-built feedback controller of the NT-MDT AFM. Images are constructed from the control signal of the $Z$-axis.

$$
C_{Y}(s)=\frac{3 \times 10^{4}}{s+1.8 \times 10^{4}} .
$$

In Figs. 9 and 10, the closed-loop frequency response functions, with the aforementioned controllers, have been plotted. It is apparent from the plots that significant damping of the resonances has been achieved.

It is worth mentioning that IRC's are defined for negative imaginary systems, i.e., systems with transfer function $G(s)$, satisfying the inequality $i(G(i \omega)-G(-i \omega)) \geq 0, \forall \omega \geq 0$, [47]. It can be checked that $G_{X X}(s),(10)$, and $G_{Y Y}(s),(11)$, satisfy the negative-imaginary property, while $G_{X X}(s) T(s)$ and $G_{Y Y}(s) T(s)$ do not. Here, the IRC has been extended to 

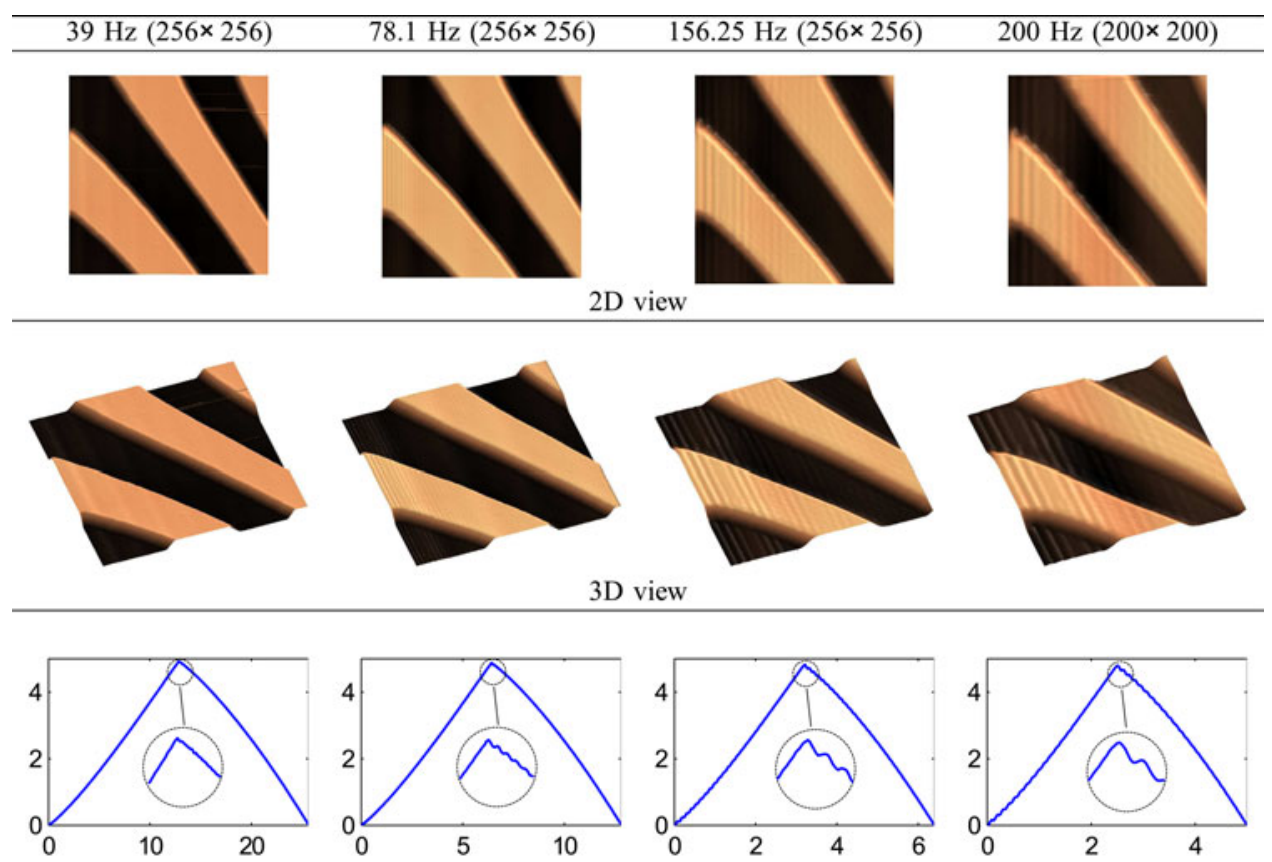

Measured X-displacement ( $\mu \mathrm{m}$ Vs msec)

Fig. 15. Open-loop scans and $X$-displacement plots.
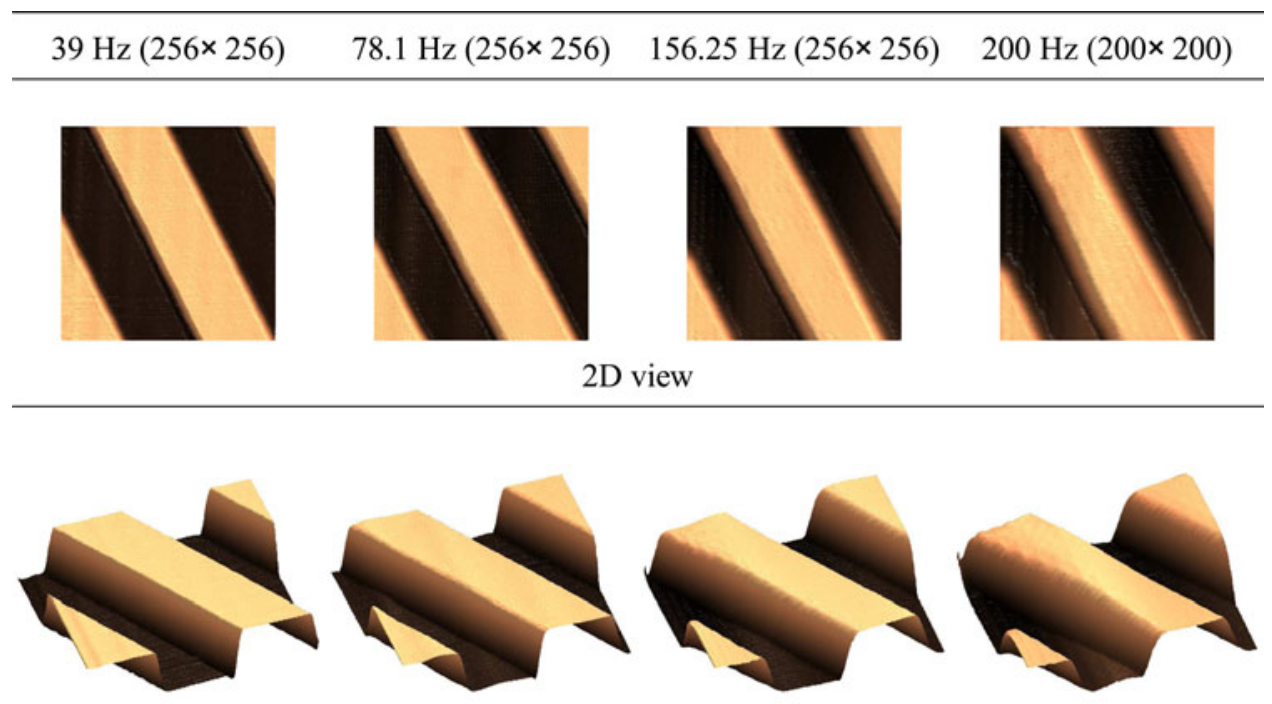

$3 \mathrm{D}$ view
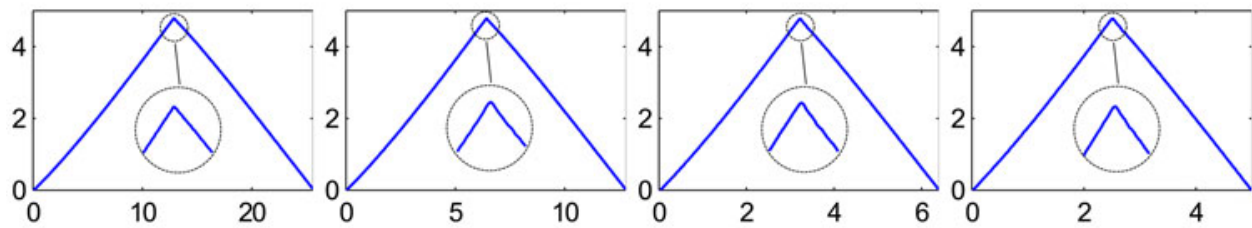

Measured X-displacement ( $\mu \mathrm{m}$ Vs msec)

Fig. 16. Closed-loop scans and $X$-displacement plots. 
accommodate the time delay. A complete stability proof, of the type give in [47], for a general negative-imaginary system with time delay is beyond the scope of this paper. However, it suffices to say that the root locus procedure presented in [14], [48] for constructing IRCs for negative-imaginary systems can be used in the current context [i.e., for $G_{X X}(s) T(s)$ and $G_{Y Y}(s) T(s)$ ].

It must be emphasized that as the system bandwidth is beyond $30 \mathrm{kHz}$, controllers (15) and (16) cannot be implemented digitally using standard rapid prototyping systems. These systems often have sampling rates that do not allow for implementation of high-bandwidth controllers. For example, dSPACE-1103 has a sampling rate of $80 \mathrm{kHz}$ that is too low for implementation of the controllers needed here. Consequently, the controllers were implemented using FPAA. An Anadigm FPAA development board (AN231K04) was used to implement the controllers. This implementation is equivalent to a direct analog implementation of the controller and allows for a much higher control bandwidth than a commercial rapid prototyping system. The analog arrays are made of switched capacitors with switching frequencies up to $15 \mathrm{MHz}$. Thus, practically eliminating the effects of sampling and quantization. Fig. 13 presents the implementation of the controller $C_{X}$, (15) in the FPAA software environment. Controller $C_{Y}$ was implemented in the same fashion. In Fig. 13, $G$ denotes the inverting unity gain $-1,-\sum$ denotes inverting summer or adder and $-\Phi$ denotes the negative of $C_{X}$. The reference signal $r_{x}$ is applied to the leads $09-10$ of the FPAA in the differential form, while the capacitive sensor output $d_{x}$ is applied to the 11-12 leads in the differential form. Thus, $n_{1}$ and $n_{3}$ in Fig. 13 denote $r_{x}$ and $d_{x}$, respectively, while $n_{2}=-\left(r_{x}-d_{x}\right)$ is the negative of the error signal and $n_{4}$ denotes the control signal to be presented to the plant. This control signal is given in its differential form along the leads $03-04$, which is converted into a single ended signal using a low-noise opamp MC33078 prior to applying it to the $X$-actuator of the nanopositioner. In the current context, $C_{X}$ can be implemented by just specifying its gain and corner frequency, which are 5 and $\left(8 \times 10^{3}\right) / 2 \pi$. A more detailed presentation on the implementation of higher order filters using FPAAs can be found in [29].

\section{AFM IMAGES}

In this section we evaluate the performance of the designed nanopositioner when used as the scanning stage of an AFM. Both closed-loop and open-loop operations of the stage are investigated and compared. The XYZ nanopositioner was installed into a commercial AFM (NT-MDT NTEGRA) to obtain images of a standard test grating. A MikroMasch TGZ2 calibration grating with $3-\mu \mathrm{m}$ period and $108-\mathrm{nm}$ step height was scanned. Scans were performed using a NT-MDT CSG30 cantilever probe with a first resonance frequency of $57 \mathrm{kHz}$ and a stiffness of $0.6 \mathrm{~N} / \mathrm{m}$. An area of $4.8 \mu \mathrm{m} \times 4.8 \mu \mathrm{m}$ of the grating was imaged at line rates of $39,78.1$, and $156.25 \mathrm{~Hz}$ at pixel resolution of $256 \times 256$. An image was also captured at $200-\mathrm{Hz}$ line rate. Due to the limitation of the data acquisition system, the resolution of images recorded at $200 \mathrm{~Hz}$ had to be reduced from 256 to 200 scan lines. Images were recorded in constant force contact mode where the vertical feedback of the microscope was activated. The calibrated sensitivity of the $Z$-axis is 253.4 $\mathrm{nm} / \mathrm{V}$. The estimated probe-sample interaction force is $60.8 \mathrm{nN}$. Topographic images of the grating were constructed from the electrical signal used to drive the $Z$-axis of the nanopositioner (see Fig. 14). When operating in closed loop, the dominant modes of the $X$ - and $Y$-axes of the nanopositioner were damped using the controller described in Section IV-A.

Oscillations were observed in the open-loop images recorded at 78.1, 156.25, and $200 \mathrm{~Hz}$. The resulting artifacts can be seen in Fig. 15. Oscillations are also clearly visible in the $X$ displacement plots measured by the corresponding displacement sensor. Fig. 16 shows that these oscillations were suppressed when the controller was activated.

Apart from oscillations, another artifact that is observed in the open-loop images is a bending of the surface features, which is partly due to the drift and the presence of hysteresis in piezoelectric actuators. The hysteresis effect can be significantly minimized, if the actuators are driven with charge sources [49], [50]. However, given that voltage amplifiers are used here, high-gain feedback can achieve a satisfactory outcome, as observed by other researchers [51]. Here, IRC control gains were chosen high enough to satisfactory hysteresis compensation.

The closed-loop images are plotted in Fig. 16. The image obtained at $78.1 \mathrm{~Hz}$ has a very similar quality as that of the 39- $\mathrm{Hz}$ image. The image quality at 156.25 and $200 \mathrm{~Hz}$ are slightly lower than that of the $39 \mathrm{~Hz}$. This is due to the limited feedback gain that can be applied to the vertical feedback loop of the microscope, which is only designed for slow scans (below $30 \mathrm{~Hz})$. An external high-gain, high-bandwidth vertical controller is currently under investigation to address this issue. Nevertheless, images with reasonable quality were successfully recorded at high speeds by using the XYZ flexure-based nanopositioner and the FPAA-based controller proposed in this paper.

\section{CONCLUSION}

In this paper, we designed a XYZ nanopositioner for highspeed AFM. FEA of the nanopositioner suggested the presence of first resonant modes at $10.7 \mathrm{kHz}$ along both the $X$ - and $Y$-axes, and $52.8 \mathrm{kHz}$ along the $Z$-axis. Experimental determination of the nanopositioner dynamics shows the presence of the first resonant modes at 8.8 and $8.9 \mathrm{kHz}$ along the $X$ - and $Y$-axes, respectively, and $48.4 \mathrm{kHz}$ along the $Z$-axis. The measured resonances are in close agreement to that of the FE model. The nanopositioner was also designed to have low cross-coupling motions among the three axes. The nanopositioner was installed on an AFM and a calibration grating was scanned, in open loop, over an area of $4.8 \mu \mathrm{m} \times 4.8 \mu \mathrm{m}$, at scanning frequencies of 39 , 78.1, 156.25, and $200 \mathrm{~Hz}$. Oscillations and the effect of hysteresis were found prominently in corresponding images. The IRC design methodology was used to realize controllers that could sufficiently damp the structural resonances and thus enable high-speed scans. Furthermore, the controller gains were chosen such that a significant reduction in hysteresis was also achieved. Due to the large bandwidth of the nanopositioner the controllers were implemented on FPAA boards, which amounts to a direct analog implementation. Scans were repeated in closed 
loop and the quality of the images were improved significantly. Almost no oscillations or hysteresis were noted.

\section{ACKNOWLEDGMENT}

The experimental research was performed at the Laboratory for Dynamics and Control of Nanosystems at the University of Newcastle, Callaghan, N.S.W., Australia.

\section{REFERENCES}

[1] U.-X. Tan, W. T. Latt, C. Y. Shee, and W. T. Ang, "A low-cost flexurebased handheld mechanism for micromanipulation," IEEE/ASME Trans. Mechatronics, vol. 16, no. 4, pp. 773-778, Aug. 2011.

[2] Y. K. Yong and T.-F. Lu, "Kinetostatic modeling of 3-RRR compliant micro-motion stages with flexure hinges," Mechanism Mach. Theory, vol. 44, no. 6, pp. 1156-1175, 2009.

[3] A. J. Fleming, "Nanopositioning system with force feedback for highperformance tracking and vibration control," IEEE/ASME Trans. Mechatronics, vol. 15, no. 3, pp. 433-447, Jun. 2010.

[4] H. C. Liaw and B. Shirinzadeh, "Neural network motion tracking control of piezo-actuated flexure-based mechanisms for micro-/nanomanipulation," IEEE/ASME Trans. Mechatronics, vol. 14, no. 5, pp. 517-527, Oct. 2009.

[5] MicroPositioning, NanoPositioning, NanoAutomation. Solutions for Cutting-Edge Technologies. Physik Instrumente Catalog, Karlsruhe, Germany, 2010.

[6] X. Sun, W. Chen, R. Zhou, J. Zhang, and W. Chen, "Design of compliant parallel mechanism for nanoimprint lithography," in Proc. 6th IEEE Conf. Ind. Electron. Appl., Jun. 2011, pp. 200-205.

[7] H. Lan, Y. Ding, H. Liu, and B. Lu, "Review of the wafer stage for nanoimprint lithography," Microelectron. Eng., vol. 84, no. 4, pp. 684688, 2007.

[8] J.-A. Kim, J. W. Kim, B. C. Park, and T. B. Eom, "Measurement of microscope calibration standards in nanometrology using a metrological atomic force microscope," Meas. Sci. Technol., vol. 17, no. 7, pp. 17921800,2006

[9] S. Polit and J. Dong, "Development of a high-bandwidth XY nanopositioning stage for high-rate micro-/nanomanufacturing," IEEE/ASME Trans. Mechatronics, vol. 16, no. 4, pp. 724 -733, Aug. 2011.

[10] T. Ando, T. Uchihashi, N. Kodera, D. Yamamoto, A. Miyagi, M. Taniguchi, and H. Yamashita, "High-speed AFM and nano-visualization of biomolecular processes," Pflügers Archiv. Eur. J. Physiol., vol. 456, no. 1, pp. 211 $225,2008$.

[11] G. Schitter, K. J. Åstrom, B. DeMartini, P. J. Thurner, K. L. Turner, and P. K. Hansma, "Design and modeling of a high-speed AFM-scanner," IEEE Trans. Control Syst. Technol., vol. 15, no. 5, pp. 906-915, Sep. 2007.

[12] Y. K. Yong and S. O. Reza Moheimani, "A compact XYZ scanner for fast atomic force microscopy in constant force contact mode," in Proc. IEEE/ASME Int. Conf. Adv. Intell. Mechatronics, Montreal, QC, Canada, Jul.2010, pp. 225-230.

[13] K. K. Leang and A. J. Fleming, "High-speed serial-kinematic AFM scanner: Design and drive considerations," Asian J. Control, vol. 11, no. 2, pp. 144-153, 2009.

[14] Y. K. Yong, S. Aphale, and S. O. R. Moheimani, "Design, identification and control of a flexure-based XY stage for fast nanoscale positioning," IEEE Trans. Nanotechnol., vol. 8, no. 1, pp. 46-54, Jan. 2009.

[15] B. J. Kenton and K. K. Leang, "Design and control of a three-axis serialkinematic high-bandwidth nanopositioner," IEEE/ASME Trans. Mechatronics, vol. 17, no. 2, pp. 1-14, Apr. 2012.

[16] A. J. Fleming, A. Wills, and S. O. R. Moheimani, "Sensor fusion for improved control of piezoelectric tube scanners," IEEE Trans. Control Syst. Technol., vol. 16, no. 6, pp. 1265-1276, Nov. 2008.

[17] T. Ando, T. Uchihashi, and T. Fukuma, "High-speed atomic force microscopy for nano-visualization of dynamic biomolecular processes," Prog. Surface Sci., vol. 83, nos. 7-9, pp. 337-437, Nov. 2008.

[18] A. Fleming, "Dual-stage vertical feedback for high-speed scanning probe microscopy," IEEE Trans. Control Syst. Technol., vol. 19, no. 1, pp. 156165, Jan. 2011.

[19] A. J. Fleming, B. J. Kenton, and K. K. Leang, "Bridging the gap between conventional and video-speed scanning probe microscopes," Ultramicroscopy, vol. 110, no. 9, pp. 1205-1214, 2010.

[20] Y. K. Yong, K. Liu, and S. O. R. Moheimani, "Reducing cross-coupling in a compliant XY nanopositioning stage for fast and accurate raster scanning,"
IEEE Trans. Control Syst. Technol., vol. 18, no. 5, pp. 1172-1179, Sep. 2010.

[21] Y. K. Yong, S. S. Aphale, and S. O. R. Moheimani, "Modeling, design and control of a fast nanopositioning stage," in Proc. IEEE/ASME Int. Conf. Adv. Intell. Mechatronics, Xi' an, China, Jul. 2-5, 2008, pp. 451-456.

[22] A. Sebastian, A. Pantazi, S. O. R. Moheimani, H. Pozidis, and E. Eleftheriou, "Achieving sub-nanometer precision in a MEMS storage device during self-servo write process," IEEE Trans. Nanotechnol., vol. 7, no. 5, pp. 586-595, Sep. 2008.

[23] Y. K. Yong, B. Arain, and S. O. R. Moheimani, "Atomic force microscopy with a 12-electrode piezoelectric tube scanner," Rev. Sci. Instrum., vol. 81, no. 3, pp. 033701-1-033701-10, 2010.

[24] I. A. Mahmood and S. O. R. Moheimani, "Making a commercial AFM more accurate and faster using positive position feedback control," Rev. Sci. Instrum., vol. 80, no. 6, pp. 063705-1-063705-8, 2009, DOI: 10.1109/TMECH.2011.2123909.

[25] B. Bhikkaji and S. O. R. Moheimani, "Integral resonant control of a piezoelectric tube actuator for fast nano-scale positioning," IEEE/ASME Trans. Mechatronics, vol. 13, no. 5, pp. 530-537, Oct. 2008.

[26] A. J. Fleming and S. O. R. Moheimani, "Sensorless vibration suppression and scan compensation for piezoelectric tube nanopositioners," IEEE Trans. Control Syst. Technol., vol. 14, no. 1, pp. 33-44, Jan. 2006.

[27] S. Aphale, A. J. Fleming, and S. O. R. Moheimani, "High speed nano-scale positioning using a piezoelectric tube actuator with active shunt control," Micro Nano Lett., vol. 2, no. 1, pp. 9-12, 2007.

[28] Y. K. Yong and S. O. R. Moheimani, "Vibration control of a novel tube scanner using piezoelectric strain-induced voltage," in Proc. IEEE/ASME Int. Conf. Adv. Int. Mechatronics, Jul. 2009, pp. 1070-1075.

[29] G. Schitter and N. Phan, "Field Programmable Analog Array (FPAA) based control of an atomic force microscope," in Proc. Amer. Control Conf., Seattle, WA, Jun. 2008, pp. 2690-2695.

[30] I. A. Mahmood and S. O. R. Moheimani, "Fast spiral-scan atomic force microscopy," Nanotechnology, vol. 20, no. 36, p. 365503, 2009.

[31] S. K. Hung, "Spiral scanning method for atomic force microscopy," in Proc. Tip Based Nanofabrication Workshop, Taipei, Taiwan, 2008, pp. 1-10.

[32] Y. K. Yong, S. O. R. Moheimani, and I. R. Petersen, "High-speed cycloid-scan atomic force microscopy," Nanotechnology, vol. 21, no. 36, p. 365503,2010

[33] Y. K. Yong and S. O. R. Moheimani, "A compact XYZ scanner for fast atomic force microscopy in constant force contact mode," in Proc. IEEE/ASME Int. Conf. Adv. Int. Mechatronics, Montreal, Canada, Jul. 6-9, 2010, pp. 225-230.

[34] B. J. Kenton and K. Leang, "Design, characterization, and control of a monolithic three-axis high-bandwidth nanopositioning stage," in Proc. Amer. Control Conf., Baltimore, MD, 2010, pp. 4949-4956.

[35] F. Beer and E. R. Johnston, Mechanics of Materials. New York: McGraw-Hill, 1992.

[36] N. Lobontiu, J. S. N. Paine, E. Garcia, and M. Goldfarb, "Corner-filleted flexure hinges," Trans. ASME, J. Mech. Des., vol. 123, pp. 346-352, 2001.

[37] N. Lobontiu, Compliant Mechanisms: Design of Flexure Hinges. Boca Raton: CRC Press, 2003

[38] N. Lobontiu and E. Garcia, "Two-axis flexure hinges with axiallycollocated and symmetric notches," Comput. Struct., vol. 81, pp. 13291341, 2003.

[39] Y. K. Yong and T.-F. Lu, "The effect of the accuracies of flexure hinge equations on the output compliances of planar micro-motion stages," Mechanism Mach. Theory, vol. 43, pp. 347-363, 2008.

[40] Y. K. Yong, T.-F. Lu, and D. C. Handley, "Review of circular flexure hinge design equations and derivation of empirical formulations," Precision Eng., vol. 32, no. 2, pp. 63-70, 2008.

[41] D. J. Inman, Engineering Vibration. Englewood Cliffs, NJ: PrenticeHall, 1996.

[42] PiezoDrive PDL200 Manual, Newcastle Innovation Ltd., Industry Development Centre, University Drive, Callaghan, N.S.W., Australia. [Online]. Available: www.piezodrive.com

[43] S. Salapaka, A. Sebastian, J. P. Cleveland, and M. V. Salapaka, "High bandwidth nanopositioner: A robust control approach," Rev. Sci. Instrum., vol. 73, no. 9, pp. 3232-3241, Sep. 2002.

[44] D. Croft, A. Sebastian, and S. Devasia, "Creep, hysteresis, and vibration compensation for piezoactuators: Atomic force microscopy application," J. Dyn. Syst., Meas., Control, vol. 123, no. 1, pp. 35-43, Mar. 2001.

[45] N. Abe and K. Yamanaka, "Smith predictor control and internal model control- a tutorial," in Proc. Soc. Instrum. and Control Eng. (SICE) Annu. Conf., 2003, vol. 2, pp. 1383-1387. 
[46] S. Aphale, A. J. Fleming, and S. O. R. Moheimani, "Integral resonant control of collocated smart structures," Smart Mater. Struct., vol. 16, no. 2, pp. 439-446, 2007.

[47] B. Bhikkaji, S. O. R. Moheimani, and I. R. Petersen, "A negative imaginary approach to modeling and control of a collocated structure," IEEE/ASME Trans. Mechatronics, [Online]. Available: http://ieeexplore.ieee.org, DOI: 10.1109/TMECH.2011.2123909, 2013.

[48] S. S. Aphale, A. J. Fleming, and S. O. R. Moheimani, "Integral resonant control of collocated smart structures," Smart Mater. Struct., vol. 16, pp. 439-446, 2007.

[49] A. J. Fleming and S. O. R. Moheimani, "A grounded load charge amplifier for reducing hysteresis in piezoelectric tube scanners," Rev. Sci. Instrum., vol. 76, no. 7, pp. 073707-073712, 2005.

[50] S. O. R. Moheimani and B. J. G. Vautier, "Resonant control of structural vibration using charge-driven piezoelectric actuators," IEEE Trans. Control Syst. Technol., vol. 13, no. 6, pp. 1021-1035, Nov. 2005.

[51] K. K. Leang and S. Devasia, "Feedback-linearized inverse feedforward for creep, hysteresis, and vibration compensation in piezoactuators," IEEE Control Syst. Technol. (Special Issue Dyn. Control Micro- and Nano-Scale Syst.), vol. 15, no. 5, pp. 927-935, Sep. 2007.

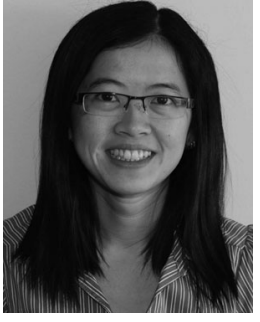

Yuen Kuan Yong (M’09) received the B.Eng. degree (Firstst Class Hons.) in mechatronic engineering and the Ph.D. degree in mechanical engineering from the University of Adelaide, Adelaide, Australia, in 2001 and 2007 , respectively.

She is currently a Postdoctoral Research Fellow at The University of Newcastle, Callaghan, Australia. Her research interests include the design and control of nanopositioning systems, highspeed atomic force microscopy, finite-element analysis of smart materials and structures, and sensing and

actuation.

Dr. Yong received the 2008 IEEE/ASME International Conference on Advanced Intelligent Mechatronics (AIM) Best Conference Paper Finalist Award. She is a member of the Technical Program Committee of AIM and the International Conference on Manipulation, Manufacturing, and Measurement on the Nanoscale (3M-NANO). She is an Editorial Board Member of the International Journal of Advanced Robotic Systems.

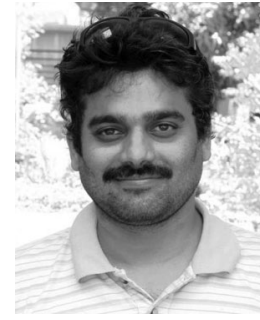

ible structures
Bharath Bhikkaji (M'09) received the Ph.D. degree in signal processing from Uppasla University, Uppsala, Sweden, in 2004

He was a Research Academic in the School of Electrical Engineering and Computer Science, University of Newcastle, Callaghan, Australia, from 2004 to 2008. He is currently an Assistant Professor in the Department of Electrical Engineering, Indian Institute of Technology Madras, Chennai, India. His research interests include system identification, robust control and active noise, and vibration control of flex-

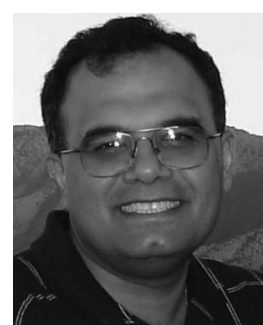

S. O. Reza Moheimani (F'11) received the Ph.D. degree in electrical engineering from the University of New South Wales at the Australian Defence Force Academy, Canberra, Australia in 1996.

He joined The University of Newcastle, Callaghan, Australia, in 1997, where he founded and directs the Laboratory for Dynamics and Control of Nanosystems, a multimillion-dollar state-of-the-art research facility. He is currently a Professor and an Australian Research Council (ARC) Future Fellow with the School of Electrical Engineering and Computer Science, The University of Newcastle. His current research interests include the area of dynamics and control at the nanometer scale, and applications of control and estimation in nanopositioning systems for high-speed scanning probe microscopy, modeling and control of microcantilever-based devices, control of microactuators in microelectromechanical systems and control issues related to ultrahigh-density probe-based data storage systems.

Prof. Moheimani is a corecipient of the 2007 IEEE TRANSACTIONS ON CONTROL Systems TeChNOLOGY Outstanding Paper Award and the 2009 IEEE CSS Control Systems Technology Award, the latter with a group of researchers from IBM Zurich Research Labs, where he has held several visiting appointments. He has served on the Editorial Boards of a number of journals including the IEEE TRANSACTIONS ON CONTROL SYSTEMS TEChNOLOGy, IEEE/ASME TRANSACTIONS ON MECHATRONICS, and Control Engineering Practice, and has chaired several international conferences and workshops. He currently chairs the IFAC Technical Committee on Mechatronic Systems. He is a Fellow of the International Federation of Automatic Control and of the Institute of Physics (U.K.) 